\title{
Access to Reproductive Healthcare Services Among African Women Living in Beijing: Understanding the Challenges
}

\author{
Mavis Adu Asuamah ${ }^{1} \cdot$ Raymond Agyenim-Boateng ${ }^{2}$
}

Received: 25 November 2021 / Revised: 21 December 2021 / Accepted: 27 December 2021 / Published online: 4 January 2022

(c) W. Montague Cobb-NMA Health Institute 2022

\begin{abstract}
A growing body of research has explored the healthcare experiences of African migrants in China. However, within this extant literature, there is a lacuna on the reproductive healthcare experiences of African women within this population. This study adopts semi-structured in-depth interviews in exploring the challenges to reproductive healthcare access among African women in Beijing. Results indicate that African women face multiple barriers to accessing reproductive healthcare. In particular, the absence of reproductive health awareness, discriminatory immigration policy, discontentment with healthcare services, and language barrier were the key challenges identified. The study highlights the challenges of reproductive healthcare experiences among African migrant women in Beijing, China, and recommends the implementation of secure and equitable policies that cater for the needs of African women and minorities in the healthcare setting.
\end{abstract}

Keywords African women $\cdot$ Reproductive health $\cdot$ Challenges $\cdot$ China

\section{Introduction}

Within the context of migration, access to healthcare services is often precarious for a population at the margins of society. Several studies have explored barriers that affect healthcare access among minority groups [1-4]. Structural factors such as language, culture, gender, financial, ethnicity, social-economic status, and a host of others have clarified the complexity of healthcare access among a vulnerable population across several different countries [5-7]. Projections are indicative that there are about 500,000 Africans in China [8]. African migrants in China fall within a vulnerable group and experience multiple barriers to healthcare services. As previously reported [9], discrimination prevents Africans in South China from accessing quality healthcare. While there are several journal articles on Africans across the cities of China $[9,10]$, the vast majority of research on African

Raymond Agyenim-Boateng

raymondowusuboateng@ln.hk

Mavis Adu Asuamah

mavisasuamah@gmail.com

1 Department of Social Policy, Beijing Normal University, Beijing, China

2 Department of Psychology, Lingnan University, Hong Kong, SAR, China migrants in China is situated on Africans in Guangzhou; the capital city of Guangdong province $[8,11]$. Indeed, Guangzhou has attracted many African traders and dwellers leading to exponential scholarly attention on the African population in this region. Despite public health; a key element of China-Africa cooperation, African migrants continue to experience various barriers to healthcare access in China $[11,12]$. Research has explored the healthcare experiences among African migrants in China [11, 13]. Within this body of research, evidence suggests that African migrants experience critical barriers to healthcare due to a combination of legal issues, language barriers, unfamiliar health settings, cultural norms, health beliefs, and a host of others [11-13]. A recent large-scale study has found depression prevalence among this population [10]. However, there is a paucity of research on the reproductive healthcare experiences among women in this population. To the extent that, not a single research has explored the reproductive healthcare experiences among African women in China. Meanwhile, it is worth mentioning that reproductive health is an integral part of the modern human rights system [14, 15]. "Reproductive health is a state of complete physical, mental and social well-being and not merely the absence of disease or infirmity, in all matters relating to the reproductive system and its functions and processes" [16]. Reproductive health demands that individuals have the agency to a satisfying and safe sex, 
reproduce and decide if, when and how often to do so [16]. Evidence from different samples has identified various factors hindering women from accessing quality reproductive healthcare services [17-19]. Yet, the available research has failed to provide the necessary distinction and consideration of demographic characteristics that compound the reproductive healthcare experiences of women in a given context. Furthermore, migrant health research in Asian countries [20] as well as the reproductive healthcare experiences of African migrants in China is sparse. Considering this gap, this study focused on reproductive healthcare experiences and factors that hinder African migrant women from accessing quality reproductive healthcare services in Beijing, China.

\section{Theoretical Framework}

Women's health status is affected by complex biological, social, and cultural factors which are interrelated and can only be addressed in a comprehensive way [15]. Understanding the challenges to reproductive healthcare requires an extensive examination of how multiple factors influence the individual's decision to reproductive healthcare participation. Accordingly, the study drew on the Anderson Behavioural Model of Health Service Utilization [21, 22] and Intersectionality [23] to develop an integrated framework to advance our understand of the reproductive healthcare experiences of African women in China. Previous research has demonstrated the usefulness of these theories in studying the healthcare experiences of minority groups $[1,11]$. Hence, these theoretical perspectives provide a useful basis to the understanding of the problems African women face in accessing reproductive healthcare. Anderson's Behavioural Model of Health Service Utilization posits three antecedents' factors (namely: predisposing factors, enabling factors, and need) that enable or impede the use and need of healthcare service. According to the model; race, age, and health beliefs make up for the predisposing factors. Family support, access to health insurance, and community are characteristics of "enabling factors". Need entails perceived and the actual need for health service [21, 22]. Essentially, these factors enable or preclude health service utilization and optimization [21, 22]. Intersectionality [23], on the other hand, offers the analytical framework to understanding how multiple factors generate different modes of advantage and discrimination. Thus, in the case of the present research, African women being a vulnerable group within a minority; a look at intersectionality analysis will help us understand the complexity of their experiences regarding reproductive healthcare. Together, incorporating these two theories into one conceptual framework enables the study to tease out the additional challenges that affect the healthcare experiences of African migrant women in China.

\section{Methods}

This study was conducted in Beijing. As already mentioned above, research on African migrants in Beijing is relatively little as compared to other cities in China [8]. Moreover, Beijing being the capital of China; serves as an interesting case for this exploration.

\section{Participants}

We conducted 15 semi-structured in-depth interviews among African women living in Beijing. The eligibility criteria were women above 22 years of age (this criterion was set to ensure that participants were mature women) with fluency in English, living in Beijing for a minimum of two years (this criterion allowed the interviews to be conducted in English and aligns with the study aim). We relied on a purposive sampling approach in attracting a pool of participants for the study. We recruited African women in Beijing through different communal societies and organizations. We reached out to the Association of African Women Diplomats and Wives, the diverse African Business Communities, and religious groups in Beijing to attract a pool of interviewees. These groups had been a very helpful source of contact. We submitted a signup sheet with a blurb to the various contact persons of those societies asking members for their help to participate in the research. Overall, a total of twenty-three women signed up and indicated their interest to participate. However, the response rate was about $80 \%$ as some of the signees were not reachable; others too could not commit to the interview.

\section{Materials and Procedure}

Considering the nature and limited research on reproductive healthcare, we employed an exploratory design to provide an in-depth understanding of African women's reproductive healthcare experiences in Beijing. The interview protocol was developed by both authors and face validated by two independent researchers and an expert interested in health policy research at the Department of Social Policy, Beijing Normal University. We conducted face-to-face semi-structured in-depth interviews using open-ended questions. Specifically, the interviews explored; participants' knowledge of reproductive health, reproductive healthcare participation, and the challenges associated with reproductive healthcare access in Beijing. The interviews were conducted in English and lasted for about 60 to 120 minutes maximum. To ensure that the interviews progressed smoothly and in a safe space, all the interviews were conducted by the first author who shared 
the same sex with the interviewees. The interviews were conducted between the month of May and July in the year 2019 right before the COVID-19 outbreak. The interviews were conducted in places of convenience, mostly at the interviewee's residence, two interviews at a church premise and two at a hair salon. No incentive was provided for participation. All the interviews were tape-recorded and later transcribed for the analysis. We ended data collection at 15 interviews as we were having repetitive data. Moreover, the recorded data were adequate to construct a valid understanding of our research aim.

\section{Ethics}

The study protocol was approved by the institutional review board of Beijing Normal University (Ref: SP012/18/19). At the beginning of the interviews, we presented an information sheet to participants explaining the research and the possibility of publishing the results in academic journals. Consent was obtained by asking each participant to indicate their consent to participate by signing a consent document. Participation was voluntary, and confidentiality was ensured. No personal details were collected except age, nationality, and occupation. To protect the confidentiality and anonymity of participants' identities and other recognizable data, the tape recordings and transcripts from the interviews were not made available to anybody not directly involved in the study.

\section{Analysis}

The data were analyzed using thematic analysis. We first transcribed all the tape-recorded interviews and read them individually without initial coding. Upon reading the transcripts on the second round, we developed open coding with a specific focus on the challenges and barriers pointed out by participants. We coded the barriers and challenges into categories and themes. The initial coding was cost, affordability, stressful birth registration, immigration issues, language issues, and discontentment with local healthcare services. Consequently, the themes were double coded and compared by the two authors to ensure consistency. In the end, we settled on four themes, namely; the lack of reproductive health awareness, discriminatory immigration policies, discontentment with health services, and language barrier.

\section{Results}

\section{Demographic Characteristics of Participants}

Demographic characteristics of participants $(N=15)$ are presented in Table 1. Participants originated from Botswana, Cameroon, Gabon, Ghana, Ivory Coast, Liberia, South Africa, Togo, Tanzanian, and Zambia. The mean age was 33.3 years with a standard deviation of 7.5. All participants reported English as their primary language of communication. The majority of the participants representing $33 \%$ $(5 / 15)$ reported being wives to a diplomat.

\section{Barriers to Reproductive Healthcare Among African Women}

The study explored the barriers that limit access to reproductive healthcare among African women in Beijing. Results from the interviews indicate that lack of reproductive health awareness, discriminatory immigration policy, discontentment with local health services, and language barrier were the key factors preventing African women from accessing quality reproductive healthcare services in Beijing.

\section{Lack of Reproductive Health Awareness}

One of the major issues hindering participants' access to reproductive healthcare was the lack of reproductive health awareness. It is a clear indication that cultural and societal norms that African women had been previously exposed to influenced their reproductive healthcare participation.

Table 1 Demographic characteristics of participants $(N=15)$

\begin{tabular}{llll}
\hline Participants & Country of origin & Age & Occupation \\
\hline 1 & Gabon & 36 & Student \\
2 & Congo & 45 & Diplomat's wife \\
3 & Togo & 29 & Hairdresser \\
4 & Ghana & 27 & Student \\
5 & Liberia & 30 & Diplomat's wife \\
6 & South Africa & 39 & Business Woman \\
7 & Ivory Coast & 37 & Trader \\
8 & Ghana & 46 & Diplomat's wife \\
9 & Tanzanian & 36 & Diplomat's wife \\
10 & Cameroon & 28 & Businesswoman \\
11 & Botswana & 20 & Student \\
12 & Zambia & 30 & Hair Dresser \\
13 & Liberia & 24 & Student \\
14 & Cameroon & 27 & Trader \\
15 & Togo & 38 & Diplomat's wife \\
\hline
\end{tabular}


Most participants outlined that reproductive healthcare is about obstetrics.

This is mostly for women...it is all about taking care of yourself very well as a woman by frequently seeing a doctor for body assessment, during, and after pregnancy. (Participant 6)

Participants also expressed that the aged woman does not need to access reproductive healthcare as they had passed pregnancy bearing age. For example, participants had this to say:

Coming from a Muslim community, it is regarded as a crime or a taboo for teachers to discuss reproductive health with students, even at the tertiary level. I remember vividly some parents had to withdraw their Children from my School while I was in Senior High School because our class teacher taught us how to take care of ourselves as adolescents. (Participant 3) I am old now, and even when I was young I was not going to the hospital specifically for my reproductive health care unless when I get pregnant, and so even now that I have no intentions to give birth again, I don't see the essence of seeing a doctor because of that. (Participant 9)

\section{Discriminatory Immigration Policies}

Other issues stemming from the institutional level emerged from the interviews. Participants outlined the difficulty associated with getting visas and birth certification for newborn African babies. All of the respondents admitted Chinese immigration system itself poses barriers to reproductive healthcare services. Participants 7 and 4 reported:

Chinese immigration laws are biased towards foreign women including me because when I gave birth in China, it was really difficult to secure a visa and birth certificate for my son. I had to travel to my country to prepare a passport and other documents before coming back. After that incident, I have decided never to give birth in China...Even all my friends too. I will travel home, deliver, and come back. (Participant 4) Chinese laws are not advanced enough to separate foreign women from being treated separately as their local counterparts; I am saying this because when I was pregnant, the doctor refused to reveal the sex of my baby because it is forbidden. This is not reasonable because if our children cannot be given Chinese citizenship, then we should be treated differently.... this phenomenon scares me from accessing reproductive healthcare in China. (Participant 7)
Responses took a different twist yet stemming from immigration policy. Issues relating to cost and financial constraints were reported to hinder participants' access to quality reproductive healthcare. Half of the participants outlined that discriminatory immigration policies concerning employment preclude African nationals from securing good-paying jobs to be able to afford reproductive healthcare. Participant 10 added:

I am educated but cannot secure a good-paying job in China because some of the skill sets are reserved for some nationals from western countries. A discriminatory practice, but because there are no mechanisms in place to correct it, some of us are left out of good jobs. I don't earn enough to spend on reproductive healthcare which is usually expensive. (Participant 10)

The following excerpts clearly show that financial constraints mostly springing from immigration policy on employment prevented participants from accessing reproductive healthcare services. Some participants expressed the willingness to attend international hospitals in Beijing but then again cited issues of cost and affordability as the major deterrents.

\section{Discontentment with Health Services}

Another challenge reported was dissatisfaction with the local health services. The majority of participants reported that the healthcare system in Beijing was not foreigner-friendly. For example, participants cited issues with childbirth, poor communication at the hospital, and difficulty getting a visa for newborn babies. Deducing from the interviews, participants equally indicated their preference for international hospitals over local Chinese hospitals for reproductive healthcare partly because of dissatisfaction with local Chinese hospitals. Participants expressed their discontentment in this manner:

My friends complain that as foreigners, the doctors treat them as Chinese and apply Chinese regulations on them. It happens to me... Imagine doctors not willing to reveal the sex of your baby until it is born. Also, if there are any serious complications, you have to force the doctor before they disclose such information.... I found that a bit strange honestly. (Participant 12) I don't know if the doctors in Beijing are afraid to give proper treatments or what... When my friend travelled to Guangzhou, she had some problems with her womb and went for surgery there. When she returned, she needed to go back again because the hospital she was referred to here in Beijing could not treat her well. So, she went back to Guangzhou and had to spend extra money for the treatment. (Participant 4) 


\section{Language Barrier}

Language barrier was most mentioned among all participants. "Language is an obstacle in China..." Participants told. "The Chinese language is one language that is difficult for me to learn and speak..." (Participant 11). Another participant narrated: "Communication becomes a nightmare whenever I attend a Chinese hospital... I have been to the hospital where the doctor did not know what to do than to ask me to go home because we could not communicate" (Participant 2). Those who could speak and understand the language or had translators on their visits expressed some degree of satisfaction. However, a participant reported that there were instances where she observed miscommunication between the doctor and the translator. She explained:

If you can speak the language you won't have an issue visiting local hospitals. My friend recommended I went with a translator on my visit to the local hospitals...I tried that one time but at a point, I felt the doctor and the translator had some miscommunication due to their demeanour and body language. (Participant 8)

\section{Discussion}

The study explored factors hindering African women's access to quality reproductive healthcare in Beijing. To our knowledge, this is one of the few studies that solely investigate reproductive healthcare experiences among African migrant women in Beijing, China. Findings indicate that lack of reproductive health awareness, discriminatory immigration policy, discontentment with local health services, and language barrier were the key challenges preventing African women from accessing quality reproductive healthcare in Beijing. Concerning intersectionality theory [23] and Anderson Behavioural Model of Health Service Utilization [21, 22] findings imply that access to reproductive healthcare is influenced by not a single factor but rather a combination of socio-cultural, institutional, and personal factors.

Culture affects health beliefs and health literacy, which then can influence healthcare experiences [15]. There is a clear indication that cultural norms and health beliefs arising from participants' backgrounds, individual, and interpersonal levels underscore African women's attitudes towards reproductive healthcare participation in Beijing. This evidence aligns with Anderson's Behavioural Model of Health Service Utilization which suggests the antecedent factors which impede the use and need of healthcare services. Generally, it is believed that women who understand the importance of reproductive healthcare do prioritize access [15]. Thus, it is not surprising that the majority of the women interviewed had little participation in reproductive healthcare. This is problematic, as per the definition of reproductive health by WHO; reproductive health exists at all stages of life [16] as opposed to participants' ideological points of view that restrict reproductive health to childbearing as evidenced by the findings.

Discrimination immigration policies present some challenges to African women in accessing reproductive healthcare. Discriminatory immigration policy reported concerning access to reproductive healthcare was related to employment restrictions on participants and difficulty in securing visas and birth certificates for newborn babies. Issues with discrimination against Africans are not new both in academic and non-academic discourse [9, 11]. However, our findings concerning discrimination were not related to race or ethnicity as previous research has it. In our study, participants reported immigration status discrimination which permits discriminatory practices in employment and the regularization of immigration status of Chinese-born African babies. In particular, this issue is related to biased immigration laws in China which restrict jobs to nationals from certain countries. It is was clearly expressed that immigration policies were not friendly to Africans who do not fall within the foreigner resident professional category (foreigners who enter China with valid visas due to work and talent introduction), thereby making it difficult for African women to engage in employment to earn extra income to afford reproductive healthcare. Although on paper, China's immigration law applies to all foreign residents, most institutions do not treat Africans the same way they treat any other foreigner. There is a lack of proper characterization of the African immigrant in regularizing their status and the ability to enjoy social and economic rights in China [24]. For example, within the current immigration law, foreigners who enter China with valid visas due to work and talent introduction are classified as foreigner resident professionals who hold permits for work. Regrettably, most African immigrants do not fit this category due to their occupational profile and their precarious statutes that make them unfit for this category [24]. On the other hand, immigration policy does not allow the smooth regularization of stay of China-born African babies. In most instances, these babies are denied the hukou status [25] and legal documentation regardless of the status of mothers. Together, these issues contribute to the challenges African women faced in accessing reproductive healthcare services in Beijing.

Results from the study indicate discontentment with reproductive healthcare services among African women in Beijing. For example, within the current Law on Health Care for Mothers and Infants in China, sex screening of fetuses is strictly forbidden. Consequently, engaging in fetal sex identification of an unborn baby is against the law and tantamount to arrest [26]. This law took effect in June 1995 in the wake 
of the rampant abortion as a result of the growing preference for boys over girls leading to a serious sex imbalance of newborn babies [27]. African women contested being subjected to this law and resisted that "Chinese doctors can not apply Chinese regulations on their bodies because they are not Chinese". Unfortunately, this law does not discriminate; it applies to everyone in China and fails to consider the implication it might have on culturally different individuals. Extending this law to African women implies dissatisfied antenatal care and a major obstacle towards the planning and preparing for baby process. African women expressed discontentment with this law and were reluctant to access gestation services (the most familiar aspect of reproductive health among participants). In effect, this law was particularly pertinent to the barriers to reproductive healthcare among African women that were interviewed.

Findings from the study indicate that African women and Chinese healthcare providers cannot communicate. Language access is yet another challenge that African women faced in navigating accessible reproductive healthcare. In addition, we found that language barrier leads to miscommunication between Chinese medical professionals and African women. The language barrier identified in our findings aligns with previous studies [1, 11, 28]. Previous research has demonstrated the association between language barrier and less satisfaction with health services between both parties. Similarly, language barrier decreases the quality of healthcare delivery and patient safety [29, 30]. Ideally, the use of health services demands effective communication between a patient and health service provider. However, healthcare facilities are far from overcoming language barriers in healthcare despite the increasing number of migrant patients. This calls for urgent attention for Chinese healthcare facilities to keep abreast of the communication needs of their diverse patients to provide better solutions.

\section{Implications and Recommendations}

In practice, we recommend that Chinese healthcare facilities offer language training for health professionals and involve professional interpreter services. Collectively, this can overcome the language barrier and better facilitate effective communication between African women and their Chinese health professionals. Again, the government of China could ease the processes involved in the acquisition of documentation such as birth certificates for China-born African babies. Moreover, the law on sex identification of fetuses should be relaxed for foreigners so that they could make informed decisions and preparations before putting to bed.

Our findings indicate illiteracy as a contributing factor hindering African women's access to quality reproductive healthcare. It is apparent that participants only taught of reproductive health as prenatal and antenatal and tended to be adamant about the regular body checks and health screening which form part of reproductive healthcare and wellbeing. Essentially, the study admonished African women to seek information on reproductive health and consider it equally as important as general health. In addition, the various diplomatic missions in Beijing could take up the responsibility to inform and educate African women to understand the delicate nature of reproductive health issues and the need for frequent body checks. In closing, our findings underscore the importance for policymakers and health institutions to utilize these recommendations in making reproductive healthcare decisions tailored for African women and other minority women in Beijing and entire China.

\section{Conclusion}

This study provides additional evidence to the expanding body of research on the challenges to healthcare access among Africans in China. Our study did so by adding a nuanced understanding of the reproductive healthcare experiences of African migrant women and highlighting the barriers these women face in accessing reproductive healthcare in Beijing, China. These challenges stem from institutional, structural, cultural, and personal factors which predispose African women from accessing quality reproductive healthcare. To ameliorate these challenges, there is the need for the implementation of integrated, secure, and equitable policies, and healthcare services tailored to the reproductive healthcare needs of the African migrant woman in China.

Despite this contribution, the study has some limitations. First, findings from this study are limited to African migrant women in Beijing owing to the study's purposive and convenience sampling approach. Second, although data reached saturation at 15 interviews, the methodological approach which requires a smaller sample size prevents us from drawing a definite conclusion. Hence, future research should adopt a robust methodology to investigate such conventions.

Author Contribution The first author conceptualized, designed the work, and did the data collection. Both authors did the analysis and interpretation. The second author prepared the manuscript for submission. Both authors have critically reviewed and approved the submission of this manuscript.

\section{Declarations}

Ethics Approval The study protocol was reviewed and approved by the institutional review board of Beijing Normal University. (Ref: SP012/18/19).

Consent to Participate In the beginning of the interviews, we presented an information sheet to participants explaining the research and the 
possibility of publishing the results in academic journals. Afterwards, consent was obtained by asking each participant to indicate their consent to participate by signing a consent document. Participation was voluntary and confidentiality was ensured. No personal details were collected except nationality and age.

\section{References}

1. Shibli H, Aharonson-Daniel L, Feder-Bubis P. Perceptions about the accessibility of healthcare services among ethnic minority women: a qualitative study among Arab Bedouins in Israel. International Journal for Equity in Health. 2021;20(1):1-9. https://doi. org/10.1016/j.jpsychores.2021.110602.

2. Wang TF, Shi L, Nie X, Zhu J. Race/ethnicity, insurance, income and access to care: the influence of health status. International Journal for Equity in Health. 2013;12(1):1-7. https://doi.org/10. 1186/1475-9276-12-29.

3. Garcia-Subirats I, Vargas I, Mogollón-Pérez AS, De Paepe P, da Silva MRF, Unger JP, Vázquez ML: Barriers in access to healthcare in countries with different health systems. A cross-sectional study in municipalities of central Colombia and north-eastern Brazil. Social Science \& Medicine 2014; 106, 204-213. https:// doi.org/10.1016/j.socscimed.2014.01.054

4. Harris B, Goudge J, Ataguba JE, McIntyre D, Nxumalo N, Jikwana $S$, Chersich $M$. Inequities in access to health care in South Africa. J Public Health Policy. 2011;32(1):S102-23. https://doi. org/10.1057/jphp.2011.35.

5. Tang K, Zhao Y, Li B, Zhang S, Lee SH. Health inequity on access to services in the ethnic minority regions of Northeastern Myanmar: a cross-sectional study. BMJ Open. 2017;7(12): e017770. https://doi.org/10.1136/bmjopen-2017-017770.

6. Wang S, Kirillova K, Lehto X. Travelers' food experience sharing on social network sites. J Travel Tour Mark. 2017;34(5):680-93. https://doi.org/10.1080/10548408.2016.1224751.

7. Memon A, Taylor K, Mohebati LM, Sundin J, Cooper M, Scanlon T, de Visser R. Perceived barriers to accessing mental health services among black and minority ethnic (BME) communities: a qualitative study in Southeast England. BMJ Open. 2016;6(11): e012337. https://doi.org/10.1136/bmjopen-2016-012337.

8. Bodomo A: Historical and contemporary perspectives on inequalities and well-being of Africans in China. Asian Ethnicity 2020; 1-16. https://doi.org/10.1080/14631369.2020.1761246

9. Hall BJ, Chen W, Latkin C, Ling L, Tucker JD. Africans in south China face social and health barriers. The Lancet. 2014;383(9925):1291-2. https://doi.org/10.1016/S0140-6736(14) 60637-1.

10. Yang F, Zou X, Xiong M, Hall BJ, Sakyi K, Ong JJ, Bodomo A, Cao H, Yang B, Wang C. Mental health on the move: an observational study to characterize post-migration depression symptoms among migrants from Sub-Saharan Africa in China. J Psychosom Res. 2021;149: 110602. https://doi.org/10.1016/j.jpsychores.2021. 110602.

11. Lin L, Brown KB, Yu F, Yang J, Wang J, Schrock JM, Wong FY. Health care experiences and perceived barriers to health care access: a qualitative study among African migrants in Guangzhou, Guangdong Province, China. J Immigr Minor Health. 2015;17(5):1509-17. https://doi.org/10.1007/s10903-014-0114-8.

12. Bodomo A, Liem A, Lin L, Hall BJ. How African migrants in China cope with barriers to health care. The Lancet Public Health. 2020;5(4): e192. https://doi.org/10.1016/S2468-2667(20)30048-7.

13. Lin L, Brown KB, Hall BJ, Yu F, Yang J, Wang J, Wong FY. Overcoming barriers to health-care access: a qualitative study among African migrants in Guangzhou. China Global public health. 2016;11(9):1135-47. https://doi.org/10.1080/17441692. 2015.1076019.

14. Freeman EW, Sammel MD, Grisso JA, Battistini M, GarciaEspagna B, Hollander L. Hot flashes in the late reproductive years: risk factors for African American and Caucasian women. J Womens Health Gend Based Med. 2001;10(1):67-76. https://doi. org/10.1089/152460901750067133.

15. Ayanore MA, Pavlova M, Groot W. Unmet reproductive health needs among women in some West African countries: a systematic review of outcome measures and determinants. Reprod Health. 2015;13:5. https://doi.org/10.1186/s12978-015-0104-x.

16. World Health Organization. (n.d). Reproductive health in the western pacific. https://www.who.int/westernpacific/health-topics/reproductive-health. Accessed 20 Aug 2021.

17. Kronfol, NM: Access and barriers to health care delivery in Arab countries: a review. EMHJ-Eastern Mediterranean Health Journal 2012; 18 (12), 1239-1246, 2012. https://apps.who.int/iris/handle/ 10665/118476. Accessed 5 Oct 2021.

18. Say L, Raine R: A systematic review of inequalities in the use of maternal health care in developing countries: examining the scale of the problem and the importance of context. Bulletin of the World Health Organization 2007; 85, 812-819. https://doi.org/ 10.2471/BLT.06.035659

19. Webber G, Spitzer D, Somrongthong R, Dat TC, Kounnavongsa $\mathrm{S}$. Facilitators and barriers to accessing reproductive health care for migrant beer promoters in Cambodia, Laos, Thailand and Vietnam: a mixed-methods study. Glob Health. 2012;8(1):1-18. https://doi.org/10.1186/1744-8603-8-21.

20. Yeoh BS: Migration and health in Asia. Routledge; 2006

21. Aday LA, Andersen R. A framework for the study of access to medical care. Health Serv Res. 1974;9(3):208.

22. Andersen, RM. "Revisiting the behavioral model and access to medical care: does it matter?" Journal of health and social behavior 1995 ; $1-10$.

23. Crenshaw K: Demarginalizing the intersection of race and sex: a black feminist critique of antidiscrimination doctrine, feminist theory and antiracist politics 1989; U. Chi. Legal f., 139.

24. Zhu G, Price R. Chinese immigration law and policy: a case of change your direction, or end up where you are heading. Colum J Asian L. 2013;26:1.

25. Zhou Y. Intercultural marriage, legal status and social belonging in China: Chinese-African couples and families in Guangzhou (Doctoral dissertation, Universität zu Köln). 2017.

26. Reuters: New Chinese law prohibits sex-screening of fetuses https://www.nytimes.com/1994/11/15/world/new-chinese-lawprohibits-sex-screening-of-fetuses.html. 1994. Accessed 7 Oct 2021

27. She W. Selective sex identification of foetus banned. China population today. 1996;13(3):15.

28. Chen J, Vargas-Bustamante A, Mortensen K, Ortega AN. Racial and ethnic disparities in health care access and utilization under the Affordable Care Act. Med Care. 2016;54(2):140. https://doi. org/10.1097/MLR.0000000000000467.

29. Al Shamsi H, Almutari AG, Al Mashrafi S. Al Kalbani, T "Implications of language barriers for healthcare: a systematic review." Oman Med J. 2020;35(2): e122. https://doi.org/10.5001/omj.2020. 40.

30. Yeo S. Language barriers and access to care. Annu Rev Nurs Res. 2004;22:59-76.

Publisher's Note Springer Nature remains neutral with regard to jurisdictional claims in published maps and institutional affiliations. 